\title{
White Matter Abnormalities and Structural Hippocampal Disconnections in Amnestic Mild Cognitive Impairment and Alzheimer's Disease
}

\author{
Jared Rowley ${ }^{1}$, Vladimir Fonov ${ }^{4}$, Ona $\mathbf{W u}^{5}$, Simon Fristed Eskildsen ${ }^{6}$, Dorothee Schoemaker ${ }^{1}$, Liyong \\ $\mathbf{W u}^{1,2}$, Sara Mohades ${ }^{1}$, Monica Shin ${ }^{1}$, Viviane Sziklas ${ }^{4}$, Laksanun Cheewakriengkrai ${ }^{1}$, Amir Shmuel ${ }^{3}$, Alain \\ Dagher $^{3}$, Serge Gauthier ${ }^{1}$, Pedro Rosa-Neto ${ }^{1,3^{*}}$, for the Alzheimer's Disease Neuroimaging Initiative
}

1 Translational Neuroimaging Laboratory, McGill Centre for Studies in Aging (MCSA), McGill University, Montreal, Quebec, Canada , 2 Department of Neurology, Xuan Wu Hospital, Capital Medical University, Beijing, China, 3 McConnell Brain Imaging Centre, Montreal Neurological Institute, McGill University, Montreal, Quebec, Canada, 4 Montreal Neurological Institute, McGill University, Montreal, QC, Canada, 5 Athinoula A. Martinos Center for Biomedical Imaging, Charlestown, Massachusetts, United States of America, 6 Center of Functionally Integrative Neuroscience, Aarhus University, Aarhus, Denmark

\begin{abstract}
The purpose of this project was to evaluate white matter degeneration and its impact on hippocampal structural connectivity in patients with amnestic mild cognitive impairment, non-amnestic mild cognitive impairment and Alzheimer's disease. We estimated white matter fractional anisotropy, mean diffusivity and hippocampal structural connectivity in two independent cohorts. The ADNI cohort included 108 subjects [ 25 cognitively normal, 21 amnestic mild cognitive impairment, 47 non-amnestic mild cognitive impairment and 15 Alzheimer's disease]. A second cohort included 34 subjects [15 cognitively normal and 19 amnestic mild cognitive impairment] recruited in Montreal. All subjects underwent clinical and neuropsychological assessment in addition to diffusion and T1 MRI. Individual fractional anisotropy and mean diffusivity maps were generated using FSL-DTIfit. In addition, hippocampal structural connectivity maps expressing the probability of connectivity between the hippocampus and cortex were generated using a pipeline based on FSL-probtrackX. Voxel-based group comparison statistics of fractional anisotropy, mean diffusivity and hippocampal structural connectivity were estimated using Tract-Based Spatial Statistics. The proportion of abnormal to total white matter volume was estimated using the total volume of the white matter skeleton. We found that in both cohorts, amnestic mild cognitive impairment patients had 27-29\% white matter volume showing higher mean diffusivity but no significant fractional anisotropy abnormalities. No fractional anisotropy or mean diffusivity differences were observed between non-amnestic mild cognitive impairment patients and cognitively normal subjects. Alzheimer's disease patients had $66.3 \%$ of normalized white matter volume with increased mean diffusivity and $54.3 \%$ of the white matter had reduced fractional anisotropy. Reduced structural connectivity was found in the hippocampal connections to temporal, inferior parietal, posterior cingulate and frontal regions only in the Alzheimer's group. The severity of white matter degeneration appears to be higher in advanced clinical stages, supporting the construct that these abnormalities are part of the pathophysiological processes of Alzheimer's disease.
\end{abstract}

Citation: Rowley J, Fonov V, Wu O, Eskildsen SF, Schoemaker D, et al. (2013) White Matter Abnormalities and Structural Hippocampal Disconnections in Amnestic Mild Cognitive Impairment and Alzheimer's Disease. PLoS ONE 8(9): e74776. doi:10.1371/journal.pone.0074776

Editor: Hideyuki Sawada, National Hospital of Utano, Japan

Received February 21, 2013; Accepted August 7, 2013; Published September 27, 2013

Copyright: $\odot 2013$ Rowley et al. This is an open-access article distributed under the terms of the Creative Commons Attribution License, which permits unrestricted use, distribution, and reproduction in any medium, provided the original author and source are credited.

Funding: The MCSA data, analysis and writing of this paper were supported by Canadian institutes of Health Research (CIHR) (MOP-11-51-31); Alzheimer's Association (NIRG-08-92090); National Nature Science Foundation of China (NSFC) (3070024); Beijing Scientific and Technological New Star Program (2007B069); Nussia \& André Aisenstadt Foundation,Fonds de la recherche en santé du Québec, and the Scotia Bank Trust. Additionally, data collection and sharing for this project was funded by the Alzheimer's disease Neuroimaging Initiative (ADNI) (National Institutes of Health Grant U01 AG024904). ADNI is funded by the National Institute on Aging, the National Institute of Biomedical Imaging and Bioengineering, and through generous contributions from the following: Alzheimer's Association, Alzheimer's Drug Discovery Foundation, BioClinica, Inc., Biogen Idec Inc., Bristol-Myers Squibb Company, Eisai Inc., Elan Pharmaceuticals, Inc., Eli Lilly and Company, F. Hoffmann-La Roche Ltd. and its affiliated company, Genentech, Inc., GE Healthcare, Innogenetics, N.V., IXICO Ltd., Janssen Alzheimer Immunotherapy Research \& Development, LLC., Johnson \& Johnson Pharmaceutical Research \& Development LLC., Medpace, Inc., Merck \& Co., Inc., Meso Scale Diagnostics, LLC., NeuroRx Research, Novartis Pharmaceuticals Corporation, Pfizer Inc., Piramal Imaging, Servier, Synarc Inc., and Takeda Pharmaceutical Company. The Canadian Institutes of Health Research is providing funds to support ADNI clinical sites in Canada. Private sector contributions are Rev November 7, 2012 facilitated by the Foundation for the National Institutes of Health (www.fnih.org). The grantee organization is the Northern California Institute for Research and Education, and the study is coordinated by the Alzheimer's Disease Cooperative Study at the University of California, San Diego. ADNI data are disseminated by the Laboratory for Neuro Imaging at the University of California, Los Angeles. This research was also supported by NIH grants P30 AG010129 and K01 AG030514. The funders had no role in study design, data collection and analysis, decision to publish, or preparation of the manuscript. 
Competing interests: The authors would like to emphasize that none of the authors have employment, consultancy, patents and products in development or marketing products competing or conflicting with the results reported in this manuscript. In contrast, the large list of potential conflicts of interest reflects a requirement from ADNI. This does not alter the authors' adherence to all the PLOS ONE policies on sharing data and materials.

*E-mail:Pedro.Rosa@mcgill.ca

\section{Introduction}

Alzheimer's disease (AD) has been conceptualized by a succession of pathophysiological events beginning with progressive extracellular accumulation of amyloid followed by a variety of neurodegenerative changes such as intracellular accumulation of neurofibrillary inclusions, brain atrophy and cell depletion [1]. In AD, neurodegenerative changes (i.e. tau hyperphosphorylation and cell depletion) follows a typical 6stage topographic pattern starting in the entorhinal cortex, propagating to the limbic cortex and subsequently to the polymodal association cortex [2]. In fact, the asymptomatic AD, mild cognitive impairment $(\mathrm{MCl})$ and dementia stages nearly correspond to the severity of $A D$ neuropathology propagation [2,3].

From pathophysiological perspective, there is a growing consensus that white matter (WM) abnormalities in $\mathrm{MCl}$ constitute an integral part of the degenerative process associated with $A D$ pathophysiology. Chen and colleagues proposed that white matter pathology, as measured in-vivo in dementia patients, may be a sign of 'anterograde Wallerian degeneration', in which gray matter pathology could be preceded by axonal dysfunction [4]. WM structural changes such as myelin breakdown, loss of myelin basic protein [5], neuroinflammation as well as abnormal axonal transport have been recognized as part of AD WM neuropathological features [6-8].

The role of WM degeneration in $A D$ has been explored in vivo with Magnetic Resonance Imaging (MRI; see review $[9,10])$. Mean diffusivity (MD) and fractional anisotropy (FA) are MRI diffusion tensor imaging (DTI) outcome measures informative of microstructural organization of water in WM compartments. High MD conveys local increase of free water diffusivity in WM, which possibly is linked to reduction in myelin content, axonal depletion or declines on extracellular matrix [11]. Low FA indicates loss of diffusion directionality, which is imposed by abnormal axonal membranes. In fact, post mortem data show a correlation between FA axonal and myelin WM contents [11]. Advances in image processing allow the estimations of WM pathways, which are derived from Bayesian mathematical models (probabilistic tractography) [12]. These techniques provide a metric to estimate the degree in which WM abnormalities disrupt long pathways connecting distinct brain regions. Thus, assessment of WM abnormalities using MRI can expand classic neuropathological approach by estimating WM structural connectivity in major WM pathways $[13,14]$.

$\mathrm{MCl}$ due to $A D$ is a condition characterized by objective cognitive deficits which minimally interfere with activities of daily living [15]. Peterson and colleagues outlined a classification of $\mathrm{MCl}$ as amnestic mild cognitive impairment $(\mathrm{aMCl})$ and non-amnestic mild cognitive impairment (naMCl), based on the predominance of memory deficits over other cognitive domains [16]. It has been established that local WM disconnections between the entorhinal cortex and hippocampus (i.e. perforant path) are involved in $A D$ and $\mathrm{MCl}$ pathophysiology, however large-scale hippocampal WM connectivity has never been systematically assessed in these populations [17,18]. Large-scale hippocampus structural connectivity indicates the severity of disconnections between limbic and polymodal association cortex. Here, we aimed to compare patterns of brain FA and MD abnormalities as well as hippocampal connectivity among aMCl, naMCl and $A D$ individuals. We hypothesized that there will be greater severity of $\mathrm{MD}$ and $\mathrm{FA}$ abnormalities in $\mathrm{AD}$. In addition, we predict disconnections on large-scale hippocampal WM networks in $\mathrm{AD}$ but not in $\mathrm{aMCl}$ or naMCl.

\section{Methods}

Two cohorts were analyzed in this study. The first cohort of $\mathrm{aMCl}$ and cognitively normal $(\mathrm{CN})$ individuals was recruited at the McGill Centre for Studies in Aging (MCSA cohort) located in Montreal, Quebec, Canada. An independent cohort of $\mathrm{CN}$, $\mathrm{MCl}$, and $\mathrm{AD}$ was obtained from Alzheimer's Disease Neuroimaging Initiative Go /2 (ADNI cohort). Informed written consent was obtained from each subject in accordance with local institutions' Research Ethic Boards (REB) [19]. The McGill University Research and Ethics committee approved these protocols.

\section{MCSA cohort data acquisition}

The McGill Centre for Studies in Aging (MCSA) staff was responsible for patient recruitment, screening and enrollment in the MCSA cohort. Patients with subjective memory complaints, substantiated by a knowledgeable informant were clinically assessed (SG). Subsequently, patients underwent a full medical, neurological examination and battery of neuropsychological tests including the standard Mini Mental State Examination (MMSE) and Rey Auditory Verbal Learning Test [20]. Diagnosis of $\mathrm{MCl}$ was achieved by a consensus in a clinical diagnosis meeting based on the Peterson criteria [16].

Age and gender matched controls enrolled in this study, referred here as $\mathrm{CN}$, were recruited by advertisements in local newspapers. CN exclusion criteria were (1) presence of current or past neurological or psychiatric condition and (2) history of memory complaints. Exclusion criteria for all subjects included a history of psychological problems, intellectual inability, past psycho stimulant drug use or brain vascular lesions on the Fluid attenuation inversion recovery (FLAIR) MRI.

MRI data was acquired on a Siemens 3T Trio MR scanner (Siemens Medical Systems, Erlangen, Germany) using a 32channel phased-array head coil. Diffusion encoding was achieved using a single-shot spin-echo echo planar sequence with twice-refocused balanced diffusion encoding gradients. 
High angular resolution reconstruction was acquired with 99 diffusion encoding and 10 resting (b0) directions, $2 \mathrm{~mm}$ isotropic voxel size, 63 slices, $b=1000 \mathrm{~s} / \mathrm{mm}^{2}$, TE $=89 \mathrm{~ms}$, TR=8.3s. A $1 \mathrm{~mm}$ isotropic resolution T1-weighted anatomical scan was also acquired ( $T R=18 \mathrm{~ms}, T E=10 \mathrm{~ms}, F A=30$ degrees). The two datasets were registered using a mutual information based algorithm [21] to remove image misregistration from echo planar induced image shifts and motion. All scans were conducted at the Montreal Neurological Institute.

\section{ADNI cohort data description}

A second dataset used in the preparation of this article was obtained from the Alzheimer's Disease Neuroimaging Initiative (ADNI) database (adni.loni.ucla.edu). The ADNI was launched in 2003 by the National Institute on Aging (NIA), the National Institute of Biomedical Imaging and Bioengineering (NIBIB), the Food and Drug Administration (FDA), private pharmaceutical companies and non-profit organizations, as a $\$ 60$ million, 5year public-private partnership. The primary goal of ADNI has been to test whether serial magnetic resonance imaging (MRI), positron emission tomography (PET), other biological markers, and clinical and neuropsychological assessment can be combined to measure the progression of mild cognitive impairment $(\mathrm{MCl})$ and early Alzheimer's disease (AD). Determination of sensitive and specific markers of very early $A D$ progression is intended to aid researchers and clinicians to develop new treatments and monitor their effectiveness, as well as lessen the time and cost of clinical trials.

The Principal Investigator of this initiative is Michael W. Weiner, MD, VA Medical Center and University of California San Francisco. ADNI is the result of efforts of many coinvestigators from a broad range of academic institutions and private corporations, and subjects have been recruited from over 50 sites across the U.S. and Canada. The initial goal of $A D N I$ was to recruit 800 subjects but $A D N I$ has been followed by ADNI-GO and ADNI-2. To date these three protocols have recruited over 1500 adults, ages 55 to 90 , to participate in the research, consisting of cognitively normal older individuals, people with early or late $\mathrm{MCl}$, and people with early $\mathrm{AD}$. The follow up duration of each group is specified in the protocols for ADNI-1, ADNI-2 and ADNI-GO. Subjects originally recruited for ADNI-1 and ADNI-GO had the option to be followed in ADNI-2. For up-to-date information, see www.adni-info.org.

From the ADNI-GO and ADNI-2 dataset, we selected all participants aged 55 to 90 years of age (inclusive) who had completed, during the course of a single visit, the following clinical, imaging and neuropsychological assessments: T1 MRI, DTI, Mini Mental State Examination (MMSE), Clinical Dementia Rating scale (CDR), Wechsler Memory Scale Logical Memory II, Alzheimer's disease assessment scale (ADAS)-cog, Rey auditory verbal learning test (RAVLT). Selected individuals were classified as $\mathrm{CN}, \mathrm{MCl}$ (divided into $\mathrm{aMCl}, \mathrm{naMCl}$ ) and $\mathrm{AD}$, on the basis of clinic-behavioral measures put forth by ADNI.

The data was acquired from 14 centers around the USA and Canada between 2010 and 2012. The scanning parameters were as follows. All diffusion images were scanned on GE 3 tesla scanners. There were 41 diffusion encoding and 5 resting (b0) directions, $1.4 \mathrm{~mm} \times 1.4 \mathrm{~mm} \times 2.7 \mathrm{~mm}$ voxel size, $b=1000$ $\mathrm{s} / \mathrm{mm}^{2}$. All scans used were already EPI-eddy current corrected by ADNI. T1 scans were acquired on the same GE 3T scanner with a $1.2 \mathrm{~mm} \times 1 \mathrm{~mm} \times 1 \mathrm{~mm}$ voxel size. Further acquisition details are available from ADNI website (ADNI-INFO.org)

\section{Clinical operational definitions}

The criteria for $\mathrm{CN}$ included an MMSE score ranging between 24-30 (inclusive), and a CDR score of 0 [22,23]. $A D N I 2$ and $A D N I G O$ define $\mathrm{MCl}$ as individuals with subjective memory complaint, an MMSE score between 24-30 (inclusive), objective memory loss as shown on scores on delayed recall of one paragraph from the Wechsler Memory Scale Logical Memory II, a CDR of 0.5 , preserved activities of daily living, and the absence of dementia [24,25].

We reclassified $A D N I M C l$ individuals in $\mathrm{aMCl}$ or naMCl as defined as $1.5 \mathrm{std}$. below $\mathrm{CN}$ on $30 \mathrm{~min}$ auditory verbal learning test (AVLT) delay recall [26]. In addition to the NINCDS/ADRDA criteria for probable $A D$, mild $A D$ dementia subjects had MMSE scores between 20-26 (inclusive) and a CDR of 1 [27].

\section{T1 processing}

Individual T1 MRIs were skull stripped [28], non-uniformity corrected [29] and registered to MNI152 space nonlinearly [30]. The T1 was then classified into grey matter, white matter, and cerebrospinal fluid with a validated automated classification algorithm [31] and subsequently automatically labeled using Automatic, Nonlinear, Imaging Matching and Anatomical Labeling (ANIMAL) [32]. Individual segmented hippocampus was used as a seed region for the hippocampal structural connectivity (HSC).

\section{DTI Processing}

FA and MD maps were generated using FSL-DTIFIT from the skull-stripped [33] eddy current corrected images in MRI native space.

\section{HSC Processing}

HSC maps were generated using a pipeline based on FSL 4.1-FDT (Figure 1). In brief (1), DTI images were skull-stripped using BET2 and Eddy current-corrected [34] ; (2) At each voxel of individual DTI images, a probability distribution of fiber direction was generated using FSL-bedpostx with a maximum of two fiber directions per voxel [35] ; (3) single voxel level probabilistic maps were generated for every voxel of the seed region using FSL-probtrackx (approx. 400 maps per region; see animation in the supplementary materials). These maps represented the probability that any given voxel in the brain was connected to a seed voxel. Finally (4) a single HSC map of the entire hippocampus was computed via the max function derived from the single voxel level maps. Thus a given voxel value in the HSC map represents the likelihood of this voxel being connected to the hippocampus. HSC maps were generated with seed regions in both the left and right hippocampus (see link https://www.youtube.com/watch? v=_ugroLj3iSg). 


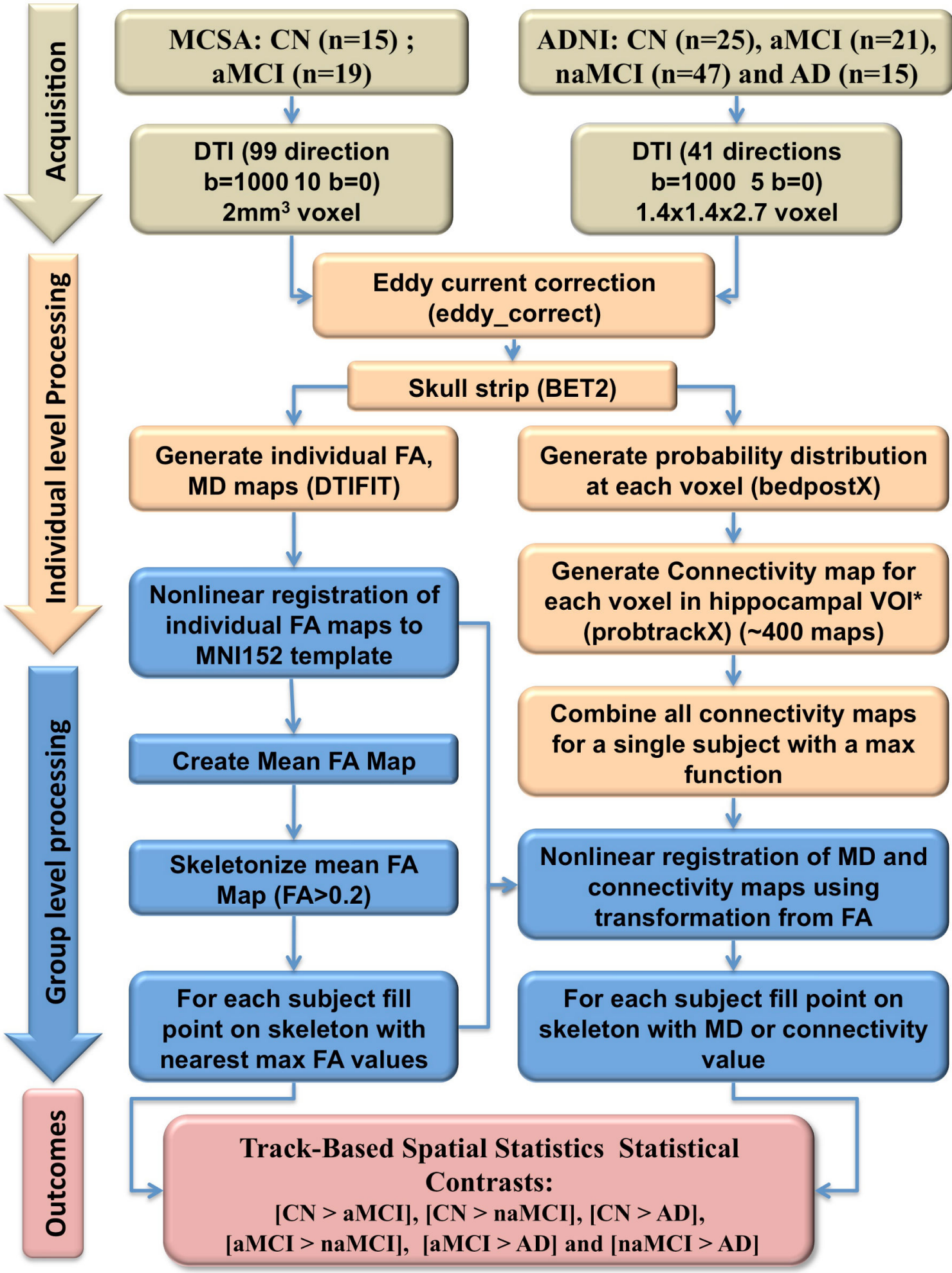

Figure 1. Summary of DTI imaging acquisition, processing and statistical outcomes. Note that DTI acquisition parameters differ for the two cohort, however, the analytical pipeline is identical for the ADNI and MCSA cohorts. Arrows indicate the flow of data through the pipeline. *Hippocampal VOI was estimated using individualized brain segmentations.

doi: 10.1371/journal.pone.0074776.g001 
Table 1. Summary of Demographic and memory scores for all groups in the ADNI and MCSA cohorts.

\begin{tabular}{|c|c|c|c|c|c|c|}
\hline & \multicolumn{2}{|c|}{ MCSACohort } & \multicolumn{4}{|c|}{ ADNI Cohort } \\
\hline & CN & aMCI & $\mathrm{CN}$ & $\mathrm{aMCl}$ & naMCl & AD \\
\hline $\mathrm{N}$ & 15 & 19 & 25 & 21 & 47 & 15 \\
\hline Gender (M/F) & $7 / 8$ & $8 / 11$ & $13 / 12$ & $12 / 9$ & $29 / 18$ & $9 / 6$ \\
\hline Age (years) & $67 \pm 5.7$ & $71.6 \pm 8.2$ & $73.3 \pm 5.5$ & $73.3 \pm 6.9$ & $73.4 \pm 7.7$ & $75.7 \pm 11.3$ \\
\hline Weight (kg) & $76 \pm 12.6$ & $64.9 \pm 12.4$ & $79.9 \pm 14.4$ & $76.3 \pm 11.6$ & $79.3 \pm 13.5$ & $76 \pm 15.9$ \\
\hline Education (years) & $15.3 \pm 2.9$ & $14.11 \pm 3.4$ & $16.2 \pm 2.7$ & $16.3 \pm 3$ & $16.2 \pm 2.6$ & $16 \pm 2.8$ \\
\hline MMSE & $29.2 \pm 1.1$ & $25.9 \pm 2.4^{*}$ & $28.9 \pm 1.5^{b, d}$ & $27.7 \pm 1.3^{\mathrm{a}, \mathrm{d}}$ & $28.2 \pm 1.5^{d}$ & $23.3 \pm 1.8^{a, b, c}$ \\
\hline RAVALT/AVLT & $9 \pm 2.1$ & $4 \pm 3^{*}$ & $12.9 \pm 1.6^{b, d}$ & $6.7 \pm 1.4^{\mathrm{a}, \mathrm{c}}$ & $12.6 \pm 1.7^{d}$ & $5.4 \pm 4.3^{a, c}$ \\
\hline APOE (carrier/n) & $1 / 8$ & $7 / 10$ & $4 / 13$ & $6 / 10$ & $15 / 41$ & $4 / 6$ \\
\hline
\end{tabular}

\section{DTI Statistics}

FA, MD and HSC statistical group differences were created using the track-based spatial statistic tool (TBSS) [36]. Firstly, all FA images were aligned to the MNI 152 standard space [37]. Then a skeleton was created from the mean FA $>0.2$. Local maxima of FA images of each subject were then projected onto the skeleton. At each voxel in the skeleton statistical group differences were determined using permutations tests (FSL randomize) [38]. MD and HSC maps were projected onto the same skeletons to determine statistical group differences. The statistical results were thickened to allow better visualization of group differences. Correction for multiple corrections was estimated using family wise error (FWE) and the threshold-free cluster enhancement (TFCE) option [38].

Global averages for FA, MD and HSC were calculated for each subject using the WM skeleton as a region of interest (ROI). In brief, for each statistical contrast comparing two groups, an FA TBSS skeleton was generated by using voxels greater than 0.2. The volume of FA skeleton was then calculated. The proportion of abnormal voxels was computed as the ratio between the volume of voxels showing statistical differences between groups and the total skeleton volume. This strategy intended to avoid the contamination grey matter signal. Regional values of FA, MD and HSC were calculated for each subject using masks based on statistical significant voxels derived from various contrast of interest. To estimate the normalized MD or FA volumes, we computed the ratio between the volume of abnormal voxels when compared to $\mathrm{CN}$ as defined by TBSS (corrected $p<0.05$ ) and the total WM skeleton volume.

In addition, in order to compare the magnitude of change across patient populations, FA, MD and HSC absolute scores were transformed as z-scores. Transformed FA, MD and HSC values were compared across groups using one-way-ANOVA.

\section{Results}

\section{Demographics}

In the MCSA cohort an unpaired one-tailed t-test showed that $\mathrm{CN}$ and aMCl groups did not differ in terms of gender, age, and educations (Table 1a). As expected, MMSE, RAVLT, and APOE status was different between groups.

In the ADNI cohort a 1-way ANOVA of the ADNI demographics showed the $\mathrm{CN}, \mathrm{aMCl}$, naMCl and $\mathrm{AD}$ groups did not differ in terms of gender, age, and education (table 1b). Post-hoc comparisons using Tukey's HSD indicated significant differences in MMSE, AVLT, and APOE status. There was no demographic difference between the aMCl group and the naMCl group other than the AVLT score.

\section{DTI outcome measures}

Global and regional FA and MD z-score values are represented in Figure 2A and 2B. Average FA and MD were significantly different between cohorts ( $F A \quad \mathrm{CN}_{\mathrm{MCSA}}=0.41$ vs $\mathrm{CN}_{\mathrm{ADNI}}=0.36$ ). The magnitude of global MD, FA and HSC abnormalities does not differ among patient groups (Figure 2A). In addition, while regional MD abnormalities predominate in aMCl, FA abnormalities are higher in AD (Figure 2B). Average HSC obtained in the $\mathrm{CN}$, depicting patterns of hippocampal connectivity known from experiments obtained in post mortem tissue, such as projections to the entire temporal neocortex as well as to cingulate cortex as well as associative parietal occipital and frontal cortices (Figure 3).

\section{DTI group differences}

In the MCSA aMCl cohort, $29 \%$ of estimated WM had significantly elevated MD compared to the $\mathrm{CN}$ group. Corpus callosum, arcuate, uncinate, superior and inferior longitudinal fascicles were affected (Figure 4A). Voxels with abnormal MD in aMCl were on average $7.1 \%$ higher than $\mathrm{CN}$. Global WM MD was elevated $5.5 \%$ in aMCl $(p=0.003)$ compared to $\mathrm{CN}$. None of the clusters of reduced FA were significant after correcting for multiple comparisons. HSC revealed no hippocampal connectivity abnormalities in aMCl. 
A

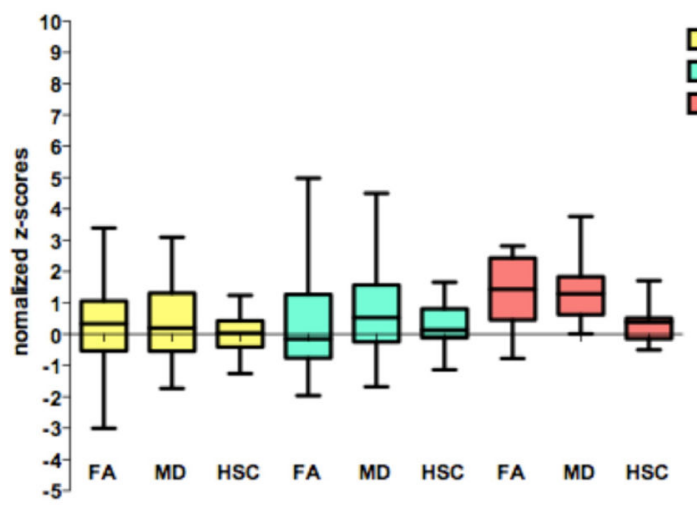

B

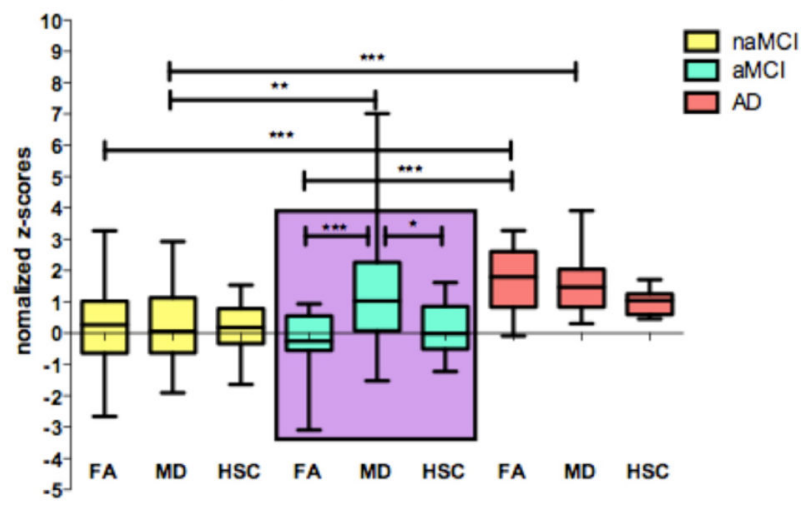

Figure 2. Global and regional magnitude of WM abnormalities in patient population. A shows that magnitude of global MD, FA and HSC abnormalities does not differ among patient groups (One way ANOVA $(p=0.0018$; $\mathrm{F}=3.212 ; \mathrm{df}=8)$. In contrast, regional $\mathrm{MD}$ abnormalities predominates in aMCl (purple box) while $\mathrm{FA}$ abnormalities arises in AD (Figure 2B -- one way ANOVA ( $p<0.0001 ; F=7.74$; $d f=8)$.

Student-Newman-Keuls Post-hoc test ${ }^{* * *}, p=0.001 ;{ }^{* *}, p=0.01$. doi: 10.1371/journal.pone.0074776.g002

In the ADNI cohort (Figure 4B) aMCl (aMCl>CN) showed abnormal mean diffusivity in $27.6 \%$ of estimated WM, particularly on the corpus callosum, splenium, superior and inferior longitudinal fascicles, and arcuate fascicles. These abnormal voxels had an average of $5.8 \%$ higher MD than $\mathrm{CN}$. Global WM MD was elevated $4.1 \%$ in aMCl compared to $\mathrm{CN}$ $(p=0.039)$ (Table 2). Similarly to the MCSA cohort, ADNI aMCI had no significant declines in FA. No WM abnormalities were observed in the reverse statistical comparison [aMCl< CN]. No hippocampal connectivity abnormalities were observed in aMCl.

In naMCI, part of ADNI cohort, neither FA nor MD values differed from $\mathrm{CN}$.

The AD patients in the ADNI cohort (Figure 4C) showed abnormally elevated MD in $66.3 \%$ of estimated WM compared to $\mathrm{CN}$. These abnormalities were observed particularly in the

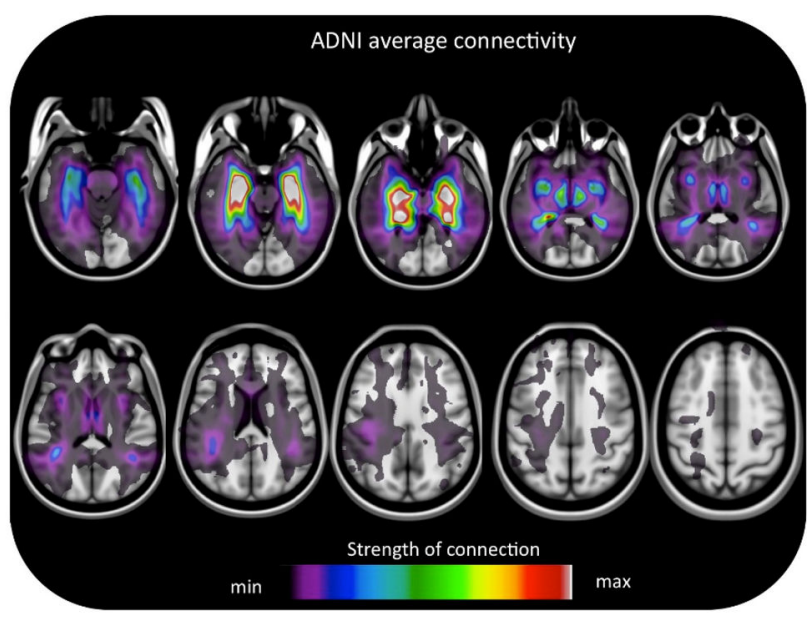

Figure 3. Hippocampal structural connectivity. Average maps expressing the hippocampal to brain structural connectivity for the $\mathrm{CN}$ group.

doi: 10.1371/journal.pone.0074776.g003

genu and splenium of the corpus callosum, uncinate, superior and inferior longitudinal fascicles and cingulate bundle. Abnormal voxels in these pathways had an average of $8.5 \%$ higher MD than CN. Global WM MD was elevated $7.7 \%$ in AD $(p=0.0001)$ (Table 2). In addition, FA was abnormally low in $54.3 \%$ of the estimated WM. In these abnormal voxels, FA was $11.3 \%$ lower than $\mathrm{CN}$. Interestingly, $75 \%$ of WM regions with abnormally lower FA also had abnormally higher MD. Global WM FA was reduced by $7.9 \%(p=0.0004)$ (Table 2$)$. No FA elevations or MD declines were observed in AD.

HSC showed reduced hippocampal connectivity (Figure $5 \mathrm{~A}$ and 5B) in the temporal lobe (angular bundle, inferior longitudinal and uncinate fascicles), limbic projections (cingulate bundle and fornix), inferior parietal cortex (arcuate fascicles) and frontal (inferior occipitofrontal and superior longitudinal fascicles) in $A D$ patients compared to $\mathrm{CN}$ (Figure 5; see link https://www.youtube.com/watch?v=LuRgO0I4TZU). All other contrasts were not significant after correcting for multiple comparisons.

The contrasts between $A D$ and naMCI patients of the ADNI cohort revealed abnormally high $\mathrm{MD}$ in $54 \%$ of $\mathrm{AD}$ estimated WMs (Figure 4D). Abnormal estimated WM in AD had an average of $7 \%$ higher MD than naMCl. Global WM MD was elevated $5.2 \%$ in $A D$ as compared to naMCl $(p=0.001)$ (Table 2). In comparison with naMCl, $A D$ patients had abnormally reduced $\mathrm{FA}$ in $57.7 \%$ of the estimated $\mathrm{WM}$. These abnormal voxels had an average of $10.6 \%$ lower $F A$ in $A D$ in comparison with naMCl. Global WM FA was reduced by $6 \%(p=0.003)$ in $\mathrm{AD}$ in comparison with naMCl (Table 2).

The $A D$ to aMCI contrast of the ADNI cohort (Figure 4A) revealed no abnormal MD voxels after correcting for multiple comparisons. Global WM MD was elevated $3.5 \%$ in $A D$ as compared to naMCI $(p=0.047)$, however this was just barely significant. FA was abnormal in $0.34 \%$ of the normalized WM voxels. Abnormal voxels had $12.5 \%$ lower FA than CN. Global WM FA was reduced by $5.3 \%(p=0.03)$ (Table 2$)$. 

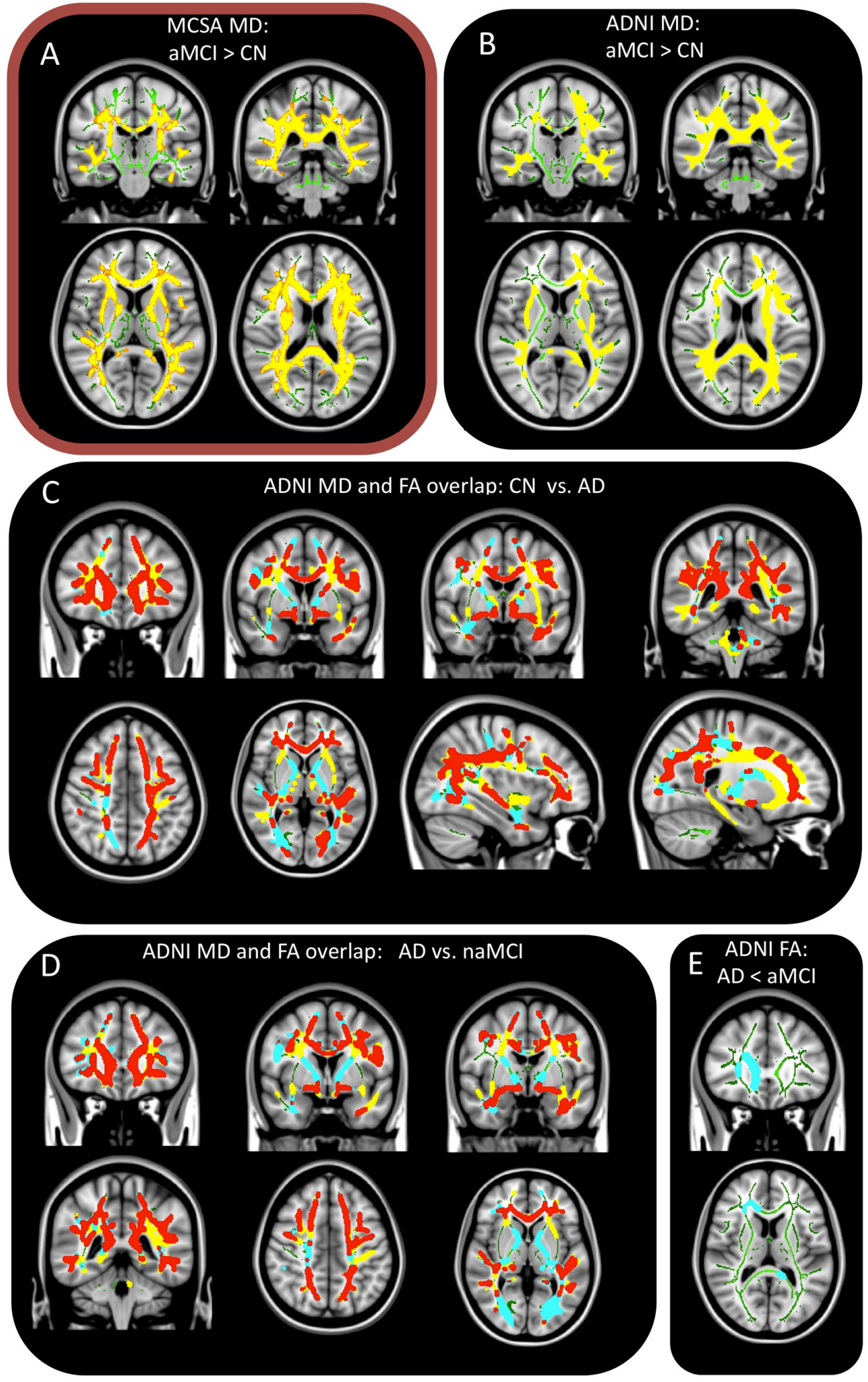

Figure 4. FA and MD group statistics. All parts display voxels showing abnormal FA and MD on TBSS skeleton. Statistically significant group differences of MD (yellow), FA (blue) are shown. A shows the $\mathrm{aMCl}>\mathrm{CN}$ contrast for the MCSA cohort while B-E show contrasts from the ADNI cohort. Notice $\mathrm{C}$ and $\mathrm{D}$ show overlap of MD and FA in red. Contrasts not shown were not significant. doi: 10.1371/journal.pone.0074776.g004 
Table 2. Summary of magnitude of global WM differences across group contrasts.

\begin{tabular}{|c|c|c|c|c|c|c|}
\hline & \multicolumn{3}{|c|}{ MCSA Cohort } & \multicolumn{3}{|c|}{ ADNI Cohort } \\
\hline & FA & MD & HSC & FA & MD & HSC \\
\hline $\mathrm{CN}$ vs. aMCl & 2.73 & $5.47^{*}$ & 12.23 & 2.45 & $4.09^{*}$ & 7.65 \\
\hline $\mathrm{CN}$ vs. naMCl & & & & 1.74 & 2.40 & 1.78 \\
\hline$C N$ vs. $A D$ & & & & $7.88^{*}$ & $7.69^{\star}$ & 9.17 \\
\hline naMCl vs. aMCl & & & & 0.70 & 1.65 & 5.97 \\
\hline naMCI vs. AD & & & & $6.04^{*}$ & $5.16^{*}$ & 7.52 \\
\hline aMCI vs. AD & & & & $5.3^{*}$ & $3.45^{*}$ & 1.65 \\
\hline $\begin{array}{l}\text { Both MCSA and } \\
\text { abnormalities are } p \\
\text { doi: } 10.1371 \text { /journa }\end{array}$ & $\begin{array}{l}\text { DNI c } \\
\text { ent in A } \\
\text { ee.007 }\end{array}$ & $\begin{array}{l}\text { ort inc } \\
\text { groups } \\
\text { 76.t002 }\end{array}$ & $\begin{array}{l}\text { ate MD } \\
0<0.05\end{array}$ & abno & ities on a & MCl. FA \\
\hline
\end{tabular}

Both FA and $M D$ values in ADNI cohort, showed no abnormalities in naMCl (aMCl vs. naMCl) after correcting for multiple comparisons.

\section{Discussion}

Brain WM forms the backbone of a large network connecting multiple segregated cortical regions, which occupies nearly as much of brain volume as the gray matter [39]. There is a growing body of the literature suggesting active WM degeneration as part of the repertoire of pathophysiological processes underlying $A D$. Although somehow neglected, the impact of WM abnormalities in $A D$ has been reported to be large $[4,11,14]$.

Here, we report increased MD (6-7\%) occurring in approximately $28 \%$ of estimated WM and no FA declines in aMCI. In contrast, the WM of AD individuals had slightly higher MD (8.5\%) and lower FA (11.3\%) affecting a larger proportion $(66 \%)$ of estimated WM. The magnitudes of MD or FA group differences reported here are above expected variability as revealed by previous DTI test-retest studies [40]. In addition, we report, for the first time, brain areas disconnected from the hippocampus due to WM abnormalities in $A D$ patients using FSL's probabilistic tractography method [12]. This technique provides the likelihood of connectivity between the hippocampus and any given brain region. Our results support the hypothesis that $A D$ brain undergoes a progressive WM degeneration characterized firstly by increased MD, followed by declines in FA and reduction of hippocampal connectivity.

The modest but significant increase of $\mathrm{MD}$ found in $\mathrm{aMCl}$ (both MCSA and ADNI cohorts) supports the construct that increased WM water diffusivity constitutes an early neurodegenerative event associated to AD pathophysiological processes. In fact, high MD has been frequently reported in aMCI populations [41-49]. In contrast using TBSS, Agosta and colleagues only found widespread changes in axial diffusivity but no significant $\mathrm{MD}$ differences in $15 \mathrm{aMCl}$ individuals, however, these disparities could be explained by methodological issues such as MRI magnetic strength as well as low in-plane resolution $[47,48,50]$.
Absence of $\mathrm{FA}$ reduction in $\mathrm{aMCl}$, as reported here, is consistent with previous studies using similar methodology (i.e. same $\mathrm{MCl}$ inclusion criteria, $3 \mathrm{~T}$ acquisitions and strict TBSS analysis) [41,42,50-52]. However, FA declines in $\mathrm{MCl}$ have been previously reported using VOI and voxel-based techniques [43,45,46,53-59]. Such discrepancy might be associated with low sample size, diagnostic criteria for $\mathrm{MCl}$, analytical methods (VOI vs. voxel-based) or correction for multiple comparisons (no correction, FDR, random field).

At least two previous studies focusing on FA and MD abnormalities in naMCl provide conflicting results $[42,60]$. In fact, older age and the presence of multiple pathologies could account for these conflicting results [60].

The two-fold increase of estimated WM showing increased $\mathrm{MD}$ in demented patients (as compared to $\mathrm{aMCl}$ ) supports the hypothesis that WM pathology also progresses in AD. Higher estimated WM showing lower FA in demented individuals in comparison with $\mathrm{aMCl}$ or naMCl further corroborates $\mathrm{WM}$ progressive degeneration in $A D$. Reduced $F A$ in $A D$ has been previously reported using $\mathrm{VOI}$ and voxel-based techniques [52,54,61-64]. [56,57,65-68] In fact, progression of WM abnormalities has been previously suggested by cross sectional and longitudinal DTI studies [41,42,51,69]. As a whole, these findings suggest increased MD as an early WM degenerative event in AD.

In post-mortem tissue, increase of tissue water diffusivity, as measured as $\mathrm{m}^{2} \mathrm{~s}^{-1}$, might occur due the reduction of tissue barriers imposed by various causes (i.e. tissue atrophy, neuroinflammation), however empirical evidence supporting this claim is undermined by the effects of tissue fixation typically utilized in post-mortem / DTI correlation studies [11]. Possibly increased $\mathrm{MD}$ in aMCI might represent an early event of WM degeneration present in AD pathophysiology secondary to the reduction of brain extracellular matrix and shrinkage of WM tissue in preclinical AD $[70,71]$.

In addition to the axonal degeneration originating due to death of cortical cell lesions, recent advances in pathophysiological mechanisms underlying FA and MD abnormalities described in $A D$ can be partially attributable to independent WM neurodegeneration. A growing body of literature suggests that WM DTI abnormalities might be secondary to neuroinflammatory factors [72]. Corroborating this hypothesis, imaging studies with PET and molecular imaging agents show an increase of microglia activation and astrocytosis in the WM of aMCl [73-75]. In addition, it is noteworthy that ventricular enlargement (a surrogate of WM atrophy) is able to accurately distinguish between $\mathrm{MCl}$ and $\mathrm{AD}$ and has been proposed as tool for measuring disease progression in the short term [76].

We also demonstrated hippocampal WM connectivity abnormalities in AD dementia. Hippocampal connectivity abnormalities affected hippocampal connections predominantly to the temporal, parietal, occipital and frontal polymodal associative areas (Figure 5). The structural connectivity outcome measure described here (HSC) represents the probability of a given brain area to be connected with the hippocampal seed region. The hippocampal seed typically encompasses all cornus amonius (CA) sectors, the dentate 

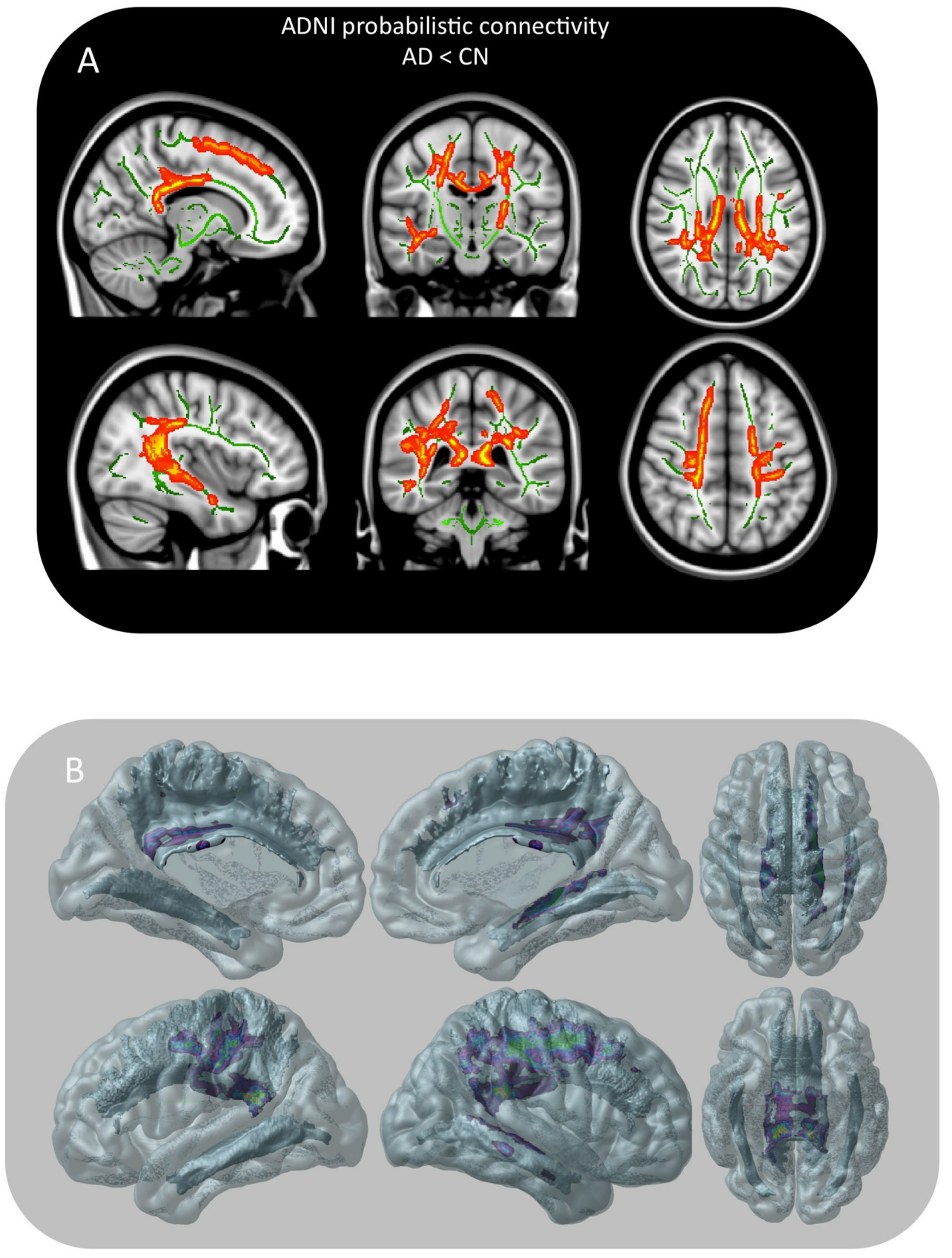

0.05 0.01

Figure 5. Hippocampal structural connectivity group statistics. Part A displays voxels showing reduction of hippocampal connectivity on the TBSS skeleton $[A D<C N]$ after correction from multiple comparisons. Note the reduction in hippocampal connectivity on angular bundle, fornix, superior longitudinal, inferior longitudinal, cingulate, uncinate and arcuate fascicles.

Part $\mathbf{B}$ shows areas of reduced connectivity in AD projected on WM pathways on a transparent brain surface space highlighting regions with reduced connectivity (See 3D animation of this image at http://www.youtube.com/watch?v=LuRgO0I4TZU). doi: 10.1371/journal.pone.0074776.g005 
gyrus and subiculum, since the limits between these areas remains below the resolution of the present DTI acquisition. For example, connectivity declines observed in the inferior occipitofrontal and superior longitudinal fascicles possibly reflect hippocampal connectivity declines mediated via subiculum, which constitutes a critical hub between the hippocampal system and the cortex [77-82]. WM areas with significant decline of probability to be connected to the hippocampus were interpreted as depleted from normal hippocampal connectivity.

The results from our HSC CN maps are in excellent agreement with the post-mortem data describing hippocampal connectivity $[83,84]$. Individual connectivity maps generated by this study in $\mathrm{CN}$ captured the classical reciprocal connections between the hippocampal system and the temporal, cingulate, inferior parietal or frontal cortices. Since the nature of DTI does not permit the inference of directionality, one cannot discriminate between hippocampal-petal and hippocampalfugal fibers. Areas with reduced structural connectivity revealed by the HSC technique in $A D$ was consistent with functional disconnections frequently reported by the literature. For example, reduced hippocampal connectivity in the cingulate bundle might explain functional disconnection reported between the hippocampus and the posterior cingulate / precuneus frequently described by numerous $A D$ rsfMRI studies $[85,86]$. Moreover, the WM connectivity reduction of the arcuate fascicles, inferior longitudinal, uncinate fascicles and superior longitudinal fascicles is a possible mechanism underling the $\left[{ }^{18} \mathrm{~F}\right] F D G$ signature of $A D$ (hypometabolism in the posterior cingulate, inferior parietal, temporal and prefrontal cortices) [87-89]. Hippocampal WM disconnections as described here corroborate the theoretical framework that emphasizes cortical disconnections as a key feature of AD [90]. In fact, neuropathological, functional neuroimaging and neuropsychological evidence indicate WM disconnections as a pathophysiological mechanism involved in $A D$.

The relative integrity of hippocampal connections in aMCl or naMCl, as predicted in our hypothesis, is supported by histopathology evidence showing tangle pathology and WM disconnection affecting predominantly transentorhinal projections to the dentate gyrus (perforant path) in aMCl, while projections from the hippocampus and subiculum and the rest of entorhinal cortex are affected in more advanced stages of the disease [2]. Possibly, a seed point in the transentorhinal cortex could better capture perforant path depletion in $\mathrm{MCl}$ stage, however transentorhinal connectivity is beyond the scope of this specific study.

\section{Limitations}

Some methodological issues limit the interpretations of the present study. Since this is a cross sectional study, inferences regarding progression of WM pathology in the spectrum of $A D$ clinical manifestations are speculative. The hypothesis posing $M D$ increases as an early WM AD pathophysiology change followed by FA declines should be assessed by appropriate longitudinal studies.

Vascular pathology is certainly a potential confounder in all studies of this nature, since vascular insults and small vessel disease constitute a frequent finding in the MRI of elderly populations. For example, white matter intensities detected in T2 or FLAIR images may have an impact on various DTI outcome measures. However, since all patients recruited in this study, had Hachinski scores lower than 4, the impact of these lesions are unlikely to be clinically significant. In contrast the impact of vascular pathology on DTI outcomes is always a limitation for dementia studies since Hachinski score $<4$ does not preclude vascular lesions. Particularly on the MCSA cohort, the presence of white matter intensities was minimal and monitored with 3D FLAIR MRI. Since the results obtained from the MCSA and ADNI cohorts were identical, it seems that vascular pathology affects these two populations in a similar fashion.

Although $A D N I$ provides a powerful database for $A D$ research, the drawback of utilizing DTI acquisitions acquired in 14 different scanners might represent a limitation for this study. While there were large efforts taken to cross-validate MRI scanners, multiple scanners acquisition is an undeniable confounding factor. Although, using the same analytical pipeline, the MCSA cohort yielded higher FA and MD control values in comparison with the same outcome measures from ADNI cohort. High FA and MD values on the MCSA cohort were obtained due to higher signal to noise ratio from the utilization a 32-channel head coil as well as 99 diffusion directions.

Regarding statistical analysis, TBSS is a conservative but extensively utilized method to compare WM change in numerous experimental populations $[42,91,92]$. Particularly in the case of this study, the results using voxel-based nonparametric statistics provide similar results to TBSS (data not shown). Analytical protocols can potentially constitute a bias particularly for those studies utilizing voxel-based parametric statistical analysis without correcting for multiple comparisons.

In conclusion, we found in aMCl WM abnormalities are characterized by high MD, which are possibly secondary to brain inflammatory changes or WM axons or myelin content depletion. Furthermore, the concomitance of $M D$ and FA abnormalities observed in $A D$ suggests higher degree of WM microstructural lesion, which impacts in large-scale brain structural connectivity. Further longitudinal studies are necessary to corroborate whether a progression of WM disease occurs in the spectrum of clinical manifestation of dementia.

\section{Acknowledgements}

Data used in preparation of this article were obtained from the Alzheimer's disease Neuroimaging Initiative (ADNI) database (adni.loni.ucla.edu). As such, the investigators within the ADNI contributed to the design and implementation of ADNI and/or provided data but did not participate in analysis or writing of this report. A complete listing of ADNI investigators can be found at: http://adni.loni.ucla.edu/wp-content/uploads/ how to apply/ADNI_Acknowledgement_List.pdf 


\section{Author Contributions}

Conceived and designed the experiments: JR PR SG. Performed the experiments: JR DS SM. Analyzed the data: JR

\section{References}

1. Jack CR Jr, Knopman DS, Jagust WJ, Shaw LM, Aisen PS et al. (2010) Hypothetical model of dynamic biomarkers of the Alzheimer's pathological cascade. Lancet Neurol 9: 119-128. doi:10.1016/ S1474-4422(09)70299-6. PubMed: 20083042.

2. Braak H, Braak E (1991) Neuropathological staging of Alzheimerrelated changes. Acta Neuropathol 82: 239-259.

3. Mufson EJ, Binder L, Counts SE, DeKosky ST, de Toledo-Morrell L et al. (2012) Mild cognitive impairment: pathology and mechanisms. Acta Neuropathol 123: 13-30. doi:10.1007/s00401-011-0884-1. PubMed: 22101321

4. Chen TF, Chen YF, Cheng TW, Hua MS, Liu HM et al. (2009) Executive dysfunction and periventricular diffusion tensor changes in amnesic mild cognitive impairment and early Alzheimer's disease. Hum Brain Mapp 30: 3826-3836. doi:10.1002/hbm.20810. PubMed: 19441023.

5. Wang DS, Bennett DA, Mufson EJ, Mattila P, Cochran E et al. (2004) Contribution of changes in ubiquitin and myelin basic protein to agerelated cognitive decline. Neurosci Res 48: 93-100. doi:10.1016/ j.neures.2003.10.002. PubMed: 14687885 .

6. Bartzokis G, Cummings JL, Sultzer D, Henderson VW, Nuechterlein KH et al. (2003) White matter structural integrity in healthy aging adults and patients with Alzheimer disease: a magnetic resonance imaging study. Arch Neurol 60: 393-398. doi:10.1001/archneur.60.3.393. PubMed: 12633151

7. Lue LF, Rydel R, Brigham EF, Yang LB, Hampel $H$ et al. (2001) Inflammatory repertoire of Alzheimer's disease and nondemented elderly microglia in vitro. Glia 35: 72-79. doi:10.1002/glia.1072. PubMed: 11424194.

8. Stokin GB, Goldstein LS (2006) Axonal transport and Alzheimer's disease. Annu Rev Biochem 75: 607-627. PubMed: 16756504.

9. Clerx L, Visser PJ, Verhey F, Aalten P (2012) New MRI Markers for Alzheimer's Disease: A Meta-Analysis of Diffusion Tensor Imaging and a Comparison with Medial Temporal Lobe Measurements. J Alzheimers Dis 29: 405-429. PubMed: 22330833.

10. Sexton CE, Mackay CE, Ebmeier KP (2009) A systematic review of diffusion tensor imaging studies in affective disorders. Biological psychiatry.

11. Gouw AA, Seewann A, Vrenken H, Van Der Flier WM, Rozemuller JM et al. (2008) Heterogeneity of white matter hyperintensities in Alzheimer's disease: post-mortem quantitative $\mathrm{MRI}$ and neuropathology. Brain 131: 3286-3298. doi:10.1093/brain/awn265. PubMed: 18927145.

12. Behrens TE, Woolrich MW, Jenkinson $M$, Johansen-Berg $H$, Nunes RG et al. (2003) Characterization and propagation of uncertainty in diffusion-weighted MR imaging. Magn Reson Med 50: 1077-1088. doi: 10.1002/mrm.10609. PubMed: 14587019

13. Charlton RA, Barrick TR, Mclntyre DJ, Shen $Y$, O'Sullivan $M$ et al. (2006) White matter damage on diffusion tensor imaging correlates with age-related cognitive decline. Neurology 66: 217-222. doi: 10.1212/01.wnl.0000194256.15247.83. PubMed: 16434657.

14. Duan JH, Wang HQ, Xu J, Lin X, Chen SQ et al. (2006) White matter damage of patients with Alzheimer, Äôs disease correlated with the decreased cognitive function. Surgical and Radiologic Anatomy 28: 150-156

15. Gauthier S, Reisberg B, Zaudig M, Petersen RC, Ritchie K et al. (2006) Mild cognitive impairment. Lancet 367: 1262-1270. doi:10.1016/ S0140-6736(06)68542-5. PubMed: 16631882.

16. Petersen RC (2004) Mild cognitive impairment as a diagnostic entity. J Intern Med 256: 183-194. doi:10.1111/j.1365-2796.2004.01388.x. PubMed: 15324362.

17. Schneider JA, Arvanitakis Z, Leurgans SE, Bennett DA (2009) The neuropathology of probable Alzheimer disease and mild cognitive impairment. Ann Neurol 66: 200-208. doi:10.1002/ana.21706. PubMed: 19743450

18. Petersen RC, Parisi JE, Dickson DW, Johnson KA, Knopman DS et al. (2006) Neuropathologic features of amnestic mild cognitive impairment. Arch Neurol 63: 665-672. doi:10.1001/archneur.63.5.665. PubMed: 16682536 .

19. Beattie BL (2007) Consent in Alzheimer's disease research: risk/benefit factors. Can J Neurol Sci 34 Suppl 1: S27-S31. PubMed: 17469678.
PR. Contributed reagents/materials/analysis tools: VF SE VS MS SM LW AS AD LC. Wrote the manuscript: JR PR SG OW SE.

20. Schmidt M (1996) Rey Auditory Verbal Learning Test: RAVLT: a Handbook: Western. Psychological Services.

21. Maes F, Collignon A, Vandermeulen D, Marchal G, Suetens P (1997) Multimodality image registration by maximization of mutual information. IEEE Trans Med Imaging 16: 187-198. PubMed: 9101328

22. Berg L (1988) Clinical dementia rating (CDR). Psychopharmacol Bull 24: 637-639. PubMed: 3249765.

23. Folstein MF, Folstein SE, McHugh PR (1975) "Mini-mental state". A practical method for grading the cognitive state of patients for the clinician. J Psychiatr Res 12: 189-198. doi: 10.1016/0022-3956(75)90026-6. PubMed: 1202204.

24. Aisen PS, Petersen RC, Donohue MC, Gamst A, Raman R et al. (2010) Clinical Core of the Alzheimer's Disease Neuroimaging Initiative: progress and plans. Alzheimers Dement 6: 239-246. doi:10.1016/j.jalz. 2010.05.778. PubMed: 20451872

25. Wechsler D (1987) WMS-R: Wechsler Memory Scale -- Revised: manual. San Antonio: Psychological Corporation.

26. Lucas JA, Ivnik RJ, Smith GE, Bohac DL, Tangalos EG et al. (1998) Mayo's older Americans normative studies: category fluency norms. J Clin Exp Neuropsychol 20: 194-200. doi:10.1076/jcen.20.2.194.1173. PubMed: 9777473.

27. Tierney MC, Fisher RH, Lewis AJ, Zorzitto ML, Snow WG et al. (1988) The NINCDS-ADRDA Work Group criteria for the clinical diagnosis of probable Alzheimer's disease A clinicopathologic study of 57 cases. Neurology 38: 359-359. doi:10.1212/WNL.38.3.359. PubMed: 3347338.

28. Eskildsen SF, Coupé P, Fonov V, Manjón JV, Leung KK et al. (2012) BEaST: Brain extraction based on nonlocal segmentation technique. Neurolmage 59: 2362-2373. doi:10.1016/j.neuroimage.2011.09.012. PubMed: 21945694

29. Sled JG, Zijdenbos AP, Evans AC (1998) A nonparametric method for automatic correction of intensity nonuniformity in MRI data. Medical Imaging, IEEE Transactions on 17: 87-97. doi:10.1109/42.668698.

30. Fonov V, Evans AC, Botteron K, Almli CR, McKinstry RC et al. (2011) Unbiased average age-appropriate atlases for pediatric studies. Neurolmage 54: 313-327. doi:10.1016/j.neuroimage.2010.07.033. PubMed: 20656036

31. Zijdenbos A, Forghani R, Evans A (1998) Automatic quantification of MS lesions in 3D MRI brain data sets: Validation of INSECT. Medical Image Computing and Computer-Assisted Interventation-MICCAl' 98. Heidelberg: Springer Verlag Berlin pp. 439-448

32. Collins DE, Evans AC (1997) ANIMAL: validation and applications of non-linear registration-based segmentation. Int J Pattern Recognit Artif Intell 11: 1271-1294. doi:10.1142/S0218001497000597.

33. Jenkinson M, Pechaud M, Smith S (2005) ET2: MR-based estimation of brain, skull and scalp surfaces.

34. Jenkinson M, Pechaud M, Smith S (2005) ET2: MR-based estimation of brain, skull and scalp surfaces pp. 12-16.

35. Behrens TE, Johansen-Berg H, Woolrich MW, Smith SM, WheelerKingshott CA et al. (2003) Non-invasive mapping of connections between human thalamus and cortex using diffusion imaging. Nat Neurosci 6: 750-757. doi:10.1038/nn1075. PubMed: 12808459.

36. Smith SM, Jenkinson M, Johansen-Berg H, Rueckert D, Nichols TE et al. (2006) Tract-based spatial statistics: voxelwise analysis of multisubject diffusion data. Neurolmage 31: 1487-1505. doi:10.1016/ j.neuroimage.2006.02.024. PubMed: 16624579 .

37. Collins DL, Neelin P, Peters TM, Evans AC (1994) Automatic 3D intersubject registration of MR volumetric data in standardized Talairach space. J Comput Assist Tomogr 18: 192-205. doi: 10.1097/00004728-199403000-00005. PubMed: 8126267

38. Nichols TE, Holmes AP (2001) Nonparametric permutation tests for functional neuroimaging: a primer with examples. Hum Brain Mapp 15: 1-25. PubMed: 11747097

39. Miller AK, Alston RL, Corsellis JA (2008) Variation with age in the volumes of grey and white matter in the cerebral hemispheres of man: measurements with an image analyser. Neuropathol Appl Neurobiol 6: 119-132. PubMed: 7374914

40. Vollmar C, O'Muircheartaigh J, Barker GJ, Symms MR, Thompson P et al. (2010) Identical, but not the same: intra-site and inter-site reproducibility of fractional anisotropy measures on two 3.0T scanners. Neuroimage 51: 1384-1394. 
41. Bosch B, Arenaza-Urquijo EM, Rami L, Sala-Llonch R, Junqué $C$ et al. (2012) Multiple DTI index analysis in normal aging, amnestic $\mathrm{MCl}$ and AD. Relationship with neuropsychological performance. Neurobiol Aging 33: 61-74. doi:10.1016/j.neurobiolaging.2010.02.004. PubMed: 20371138.

42. O'Dwyer L, Lamberton F, Bokde AL, Ewers M, Faluyi YO et al. (2011) Multiple indices of diffusion identifies white matter damage in mild cognitive impairment and Alzheimer's disease. PLOS ONE 6: e21745. doi:10.1371/journal.pone.0021745. PubMed: 21738785.

43. Cho H, Yang DW, Shon YM, Kim BS, Kim YI et al. (2008) Abnormal Integrity of Corticocortical Tracts in Mild Cognitive Impairment: A Diffusion Tensor Imaging Study. J Korean Med Sci 23: 477-483. doi: 10.3346/jkms.2008.23.3.477. PubMed: 18583886.

44. Chua TC, Wen W, Slavin MJ, Sachdev PS (2008) Diffusion tensor imaging in mild cognitive impairment and Alzheimer's disease: a review. Curr Opin Neurol 21: 83-92. doi:10.1097/WCO. Ob013e3282f4594b. PubMed: 18180656.

45. Fellgiebel A, Wille $P, M$ uuml Iler MJ, Winterer $G$, Scheurich $A$, et al (2004) Ultrastructural Hippocampal and White Matter Alterations in Mild Cognitive Impairment: A Diffusion Tensor Imaging Study. Dement Geriatr Cogn Disord 18: 101-108. doi:10.1159/000077817. PubMed: 15087585.

46. Rose SE, Chen F, Chalk JB, Zelaya FO, Strugnell WE et al. (2000) Loss of connectivity in Alzheimer's disease: an evaluation of white matter tract integrity with colour coded MR diffusion tensor imaging. J Neurol Neurosur Ps 69: 528-530. doi:10.1136/jnnp.69.4.528. PubMed: 10990518.

47. Good CD, Scahill RI, Fox NC, Ashburner J, Friston KJ et al. (2002) Automatic Differentiation of Anatomical Patterns in the Human Brain: Validation with Studies of Degenerative Dementias. Neurolmage 17: 29-46. doi:10.1006/nimg.2002.1202. PubMed: 12482066

48. Good CD (2001) A Voxel-Based Morphometric Study of Ageing in 465 Normal Adult Human Brains. Neurolmage 14: 21-36. doi:10.1006/nimg. 2001.0786. PubMed: 11525331.

49. Yasmin H, Nakata $\mathrm{Y}$, Aoki S, Abe O, Sato $\mathrm{N}$ et al. (2008) Diffusion abnormalities of the uncinate fasciculus in Alzheimer's disease: diffusion tensor tract-specific analysis using a new method to measure the core of the tract. Neuroradiology 50: 293-299. doi:10.1007/ s00234-007-0353-7. PubMed: 18246334

50. Agosta F, Pievani M, Sala S, Geroldi C, Galluzzi S et al. (2011) White matter damage in Alzheimer disease and its relationship to gray matter atrophy. Radiology 258: 853-863. doi:10.1148/radiol.10101284. PubMed: 21177393.

51. Douaud G, Jbabdi S, Behrens TE, Menke RA, Gass A et al. (2011) DTI measures in crossing-fibre areas: increased diffusion anisotropy reveals early white matter alteration in $\mathrm{MCl}$ and mild Alzheimer's disease. Neurolmage 55: 880-890. doi:10.1016/j.neuroimage. 2010.12.008. PubMed: 21182970.

52. Fellgiebel A, Wille P, Müller MJ, Winterer G, Scheurich A et al. (2004) Ultrastructural hippocampal and white matter alterations in mild cognitive impairment: a diffusion tensor imaging study. Dement Geriatr Cogn Disord 18: 101-108. doi:10.1159/000077817. PubMed: 15087585

53. Chua TC, Wen W, Chen X, Kochan N, Slavin MJ et al. (2009) Diffusion tensor imaging of the posterior cingulate is a useful biomarker of mild cognitive impairment. The American journal of geriatric psychiatry: official journal of the American Association for Geriatric Psychiatry 17: 602-613. doi:10.1097/JGP.0b013e3181a76e0b. PubMed: 19546655

54. Kiuchi K, Morikawa M, Taoka T, Nagashima T, Yamauchi T et al. (2009) Abnormalities of the uncinate fasciculus and posterior cingulate fasciculus in mild cognitive impairment and early Alzheimer\&apos;s disease: A diffusion tensor tractography study. Brain Res 1287: 184-191. doi:10.1016/j.brainres.2009.06.052. PubMed: 19559010.

55. Medina D, DeToledo-Morrell L, Urresta F, Gabrieli JD, Moseley M et al. (2006) White matter changes in mild cognitive impairment and AD: A diffusion tensor imaging study. Neurobiol Aging 27: 663-672. doi: 10.1016/j.neurobiolaging.2005.03.026. PubMed: 16005548

56. Parente DB, Gasparetto EL, da Cruz LC Jr, Domingues RC, Baptista AC, et al. (2008) Potential role of diffusion tensor MRI in the differential diagnosis of mild cognitive impairment and Alzheimer's disease. American Journal of Roentgenology 190: 1369-1374. doi:10.2214/AJR. 07.2617. PubMed: 18430857. doi:10.2214/AJR.07.2617 PubMed: 18430857

57. Stahl R, Dietrich O, Teipel SJ, Hampel H, Reiser MF et al. (2007) White Matter Damage in Alzheimer Disease and Mild Cognitive Impairment: Assessment with Diffusion-Tensor MR Imaging and Parallel Imaging Techniques. Radiology 243: 483-492. doi:10.1148/radiol.2432051714. PubMed: 17456872
58. Ukmar M, Makuc E, Onor ML, Garbin G, Trevisiol M et al. (2008) Risonanza magnetica con tensori di diffusione nella valutazione delle alterazioni della sostanza bianca nei pazienti con malattia di Alzheimer e nei pazienti con mild cognitive impairment. Radiol Med 113: 915-922. doi:10.1007/s11547-008-0286-1. PubMed: 18618077.

59. Zhang Y, Schuff N, Jahng GH, Bayne W, Mori S et al. (2007) Diffusion tensor imaging of cingulum fibers in mild cognitive impairment and Alzheimer disease. Neurology 68: 13-19. doi:10.1212/01.wnl. 0000259403.31527.ef. PubMed: 17200485.

60. Zhuang L, Wen W, Zhu W, Trollor J, Kochan N et al. (2010) White matter integrity in mild cognitive impairment: a tract-based spatial statistics study. Neuroimage 53: 16-25. doi:10.1016/j.neuroimage. 2010.05.068. PubMed: 20595067.

61. Bozzali M, Falini A, Franceschi M, Cercignani M, Zuffi M et al. (2002) White matter damage in Alzheimer's disease assessed in vivo using diffusion tensor magnetic resonance imaging. J Neurol Neurosurg, Psychiatr 72: 742-746. doi:10.1136/jnnp.72.6.742. PubMed: 12023417.

62. Choi SJ (2005) Diffusion Tensor Imaging of Frontal White Matter Microstructure in Early Alzheimer\&apos;s Disease: A Preliminary Study. J Geriatr Psychiatry Neurol 18: 12-19. doi: 10.1177/0891988704271763. PubMed: 15681623

63. Ibrahim I, Horacek J, Bartos A, Hajek M, Ripova D et al. (2009) Combination of voxel based morphometry and diffusion tensor imaging in patients with Alzheimer\&apos;s disease. Neuro endocrinology letters 30: $39-45$.

64. Medina D, Detoledo-Morrell L, Urresta F, Gabrieli JDE, Moseley M et al. (2006) White matter changes in mild cognitive impairment and AD: A diffusion tensor imaging study. Neurobiol Aging 27: 663-672. doi: 10.1016/j.neurobiolaging.2005.03.026. PubMed: 16005548.

65. Stricker NH, Schweinsburg BC, Delano-Wood L, Wierenga CE, Bangen $\mathrm{KJ}$ et al. (2009) Decreased white matter integrity in late-myelinating fiber pathways in Alzheimer's disease supports retrogenesis. Neurolmage 45: 10-16. doi:10.1016/j.neuroimage.2008.11.027. PubMed: 19100839.

66. Takahashi E, Ohki K, Kim DS (2007) Diffusion tensor studies dissociated two fronto-temporal pathways in the human memory system. Neurolmage 34: 827-838. doi:10.1016/j.neuroimage. 2006.10.009. PubMed: 17123836.

67. Taoka T, Iwasaki S, Sakamoto M, Nakagawa H, Fukusumi A et al. (2006) Diffusion anisotropy and diffusivity of white matter tracts within the temporal stem in Alzheimer disease: evaluation of the \&quot; tract of interest\&quot; by diffusion tensor tractography. AJNR Am J Neuroradiol 27: 1040-1045. PubMed: 16687540.

68. Teipel SJ, Stahl R, Dietrich O, Schoenberg SO, Perneczky R et al. (2007) Multivariate network analysis of fiber tract integrity in Alzheimer's disease. Neurolmage 34: 985-995. doi:10.1016/ j.neuroimage.2006.07.047. PubMed: 17166745 .

69. Salat DH, Tuch DS, van der Kouwe AJ, Greve DN, Pappu V et al. (2010) White matter pathology isolates the hippocampal formation in Alzheimer's disease. Neurobiol Aging 31: 244-256. doi:10.1016/ j.neurobiolaging.2008.03.013. PubMed: 18455835

70. Brückner G, Hausen D, Härtig W, Drlicek M, Arendt T et al. (1999) Cortical areas abundant in extracellular matrix chondroitin sulphate proteoglycans are less affected by cytoskeletal changes in Alzheimer's disease. Neuroscience 92: 791-805. doi:10.1016/ S0306-4522(99)00071-8. PubMed: 10426522.

71. de la Monte SM (1989) Quantitation of cerebral atrophy in preclinical and end-stage alzheimer's disease. Ann Neurol 25: 450-459. doi: 10.1002/ana.410250506. PubMed: 2774485.

72. Krstic D, Knuesel I (2012) Deciphering the mechanism underlying lateonset Alzheimer disease. Nat. Rev Neurol.

73. Cagnin A, Kassiou M, Meikle SR, Banati RB (2006) In vivo evidence for microglial activation in neuro degenerative dementia. Acta Neurol Scand: 107-114.

74. Okello A, Edison P, Archer HA, Turkheimer FE, Kennedy J et al. (2009) Microglial activation and amyloid deposition in mild cognitive impairment: a PET study. Neurology 72: 56-62. doi:10.1212/01.wnl. 0000345004.84188.b9. PubMed: 19122031.

75. Carter SF, Schöll M, Almkvist O, Wall A, Engler H et al. (2012) Evidence for astrocytosis in prodromal Alzheimer disease provided by 11C-deuterium-L-deprenyl: a multitracer PET paradigm combining 11CPittsburgh compound B and 18F-FDG. J Nucl Med 53: 37-46. doi: 10.2967/jnumed.110.087031. PubMed: 22213821

76. Nestor SM, Rupsingh R, Borrie M, Smith M, Accomazzi V et al. (2008) Ventricular enlargement as a possible measure of Alzheimer's disease progression validated using the Alzheimer's Disease Neuroimaging Initiative database. Brain 131: 2443-2454. doi:10.1093/brain/awn146. PubMed: 18669512. 
77. Goldman-Rakic PS, Selemon LD, Schwartz ML (1984) Dual pathways connecting the dorsolateral prefrontal cortex with the hippocampal formation and parahippocampal cortex in the rhesus monkey. Neuroscience 12: 719-743. doi:10.1016/0306-4522(84)90166-0. PubMed: 6472617.

78. Augustinack JC, Magnain C, Reuter M, van der Kouwe AJW, Boas D et al. (2013) MRI Parcellation of Ex Vivo Medial Temporal Lobe. Neurolmage. PubMed: 23702414

79. Barbas H, Blatt GJ (1995) Topographically specific hippocampal projections target functionally distinct prefrontal areas in the rhesus monkey. Hippocampus 5: 511-533. doi:10.1002/hipo.450050604. PubMed: 8646279.

80. Blatt GJ, Rosene DL (1998) Organization of direct hippocampal efferent projections to the cerebral cortex of the rhesus monkey: projections from CA1, prosubiculum, and subiculum to the temporal lobe. J Comp Neurol 392: 92-114. doi:10.1002/(SICI)1096-9861(19980302)392:1. PubMed: 9482235

81. Morris R, Pandya DN, Petrides M (1999) Fiber system linking the middorsolateral frontal cortex with the retrosplenial/presubicular region in the rhesus monkey. J Comp Neurol 407: 183-192. doi:10.1002/ (SICl)1096-9861(19990503)407:2. PubMed: 10213090.

82. Seltzer B, Van Hoesen GW (1979) A direct inferior parietal lobule projection to the presubiculum in the rhesus monkey. Brain Res 179: 157-161. doi:10.1016/0006-8993(79)90499-2. PubMed: 116714.

83. Insausti R, Amaral DG (2004) Insausti: Hippocampal formation Google Scholar. The human nervous system.

84. Duvernoy HM (2005) The human hippocampus: functional anatomy, vascularization and serial sections with MRI. Springer Verlag.

85. Wang K, Liang M, Wang L, Tian L, Zhang X et al. (2006) Altered functional connectivity in early Alzheimer's disease: A resting-state fMRI study. Hum Brain Mapp 28: 967-978.
86. Wang L, Zang Y, He Y, Liang M, Zhang X et al. (2006) Changes in hippocampal connectivity in the early stages of Alzheimer's disease: evidence from resting state fMRI. Neurolmage 31: 496-504. doi: 10.1016/j.neuroimage.2005.12.033. PubMed: 16473024.

87. Herholz K, Salmon E, Perani D, Baron JC, Holthoff V et al. (2002) Discrimination between Alzheimer dementia and controls by automated analysis of multicenter FDG PET. Neurolmage 17: 302-316. doi: 10.1006/nimg.2002.1208. PubMed: 12482085.

88. Villain N, Desgranges B, Viader F, De La Sayette V, Mézenge F et al (2008) Relationships between hippocampal atrophy, white matter disruption, and gray matter hypometabolism in Alzheimer's disease. J Neurosci 28: 6174-6181. doi:10.1523/JNEUROSCI.1392-08.2008. PubMed: 18550759

89. Villain N, Fouquet M, Baron JC, Mézenge F, Landeau B et al. (2010) Sequential relationships between grey matter and white matter atrophy and brain metabolic abnormalities in early Alzheimer's disease. Brain 133: 3301-3314. doi:10.1093/brain/awq203. PubMed: 20688814

90. Delbeuck X, Van der Linden M, Collette F (2003) Alzheimer'Disease as a Disconnection Syndrome? Neuropsychol Rev 13: 79-92. doi: 10.1023/A:1023832305702. PubMed: 12887040.

91. Giorgio A, Watkins KE, Chadwick M, James S, Winmill L et al. (2010) Longitudinal changes in grey and white matter during adolescence. Neuroimage 49: 94-103. doi:10.1016/j.neuroimage.2009.08.003. PubMed: 19679191.

92. Roosendaal SD, Geurts JJ, Vrenken H, Hulst HE, Cover KS et al. (2009) Regional DTI differences in multiple sclerosis patients. Neuroimage 44: 1397-1403. doi:10.1016/j.neuroimage.2008.10.026. PubMed: 19027076. 